九州大学学術情報リポジトリ

Kyushu University Institutional Repository

\title{
Napier's Constant, Imaginary Unit, Circular Constant and Some Natural Phenomena
}

Shimojo, Masataka

Laboratory of Regulation in Metabolism and Behavior, Division of Animal and Bioresource

Sciences, Department of Bioresource Sciences, Faculty of Agriculture, Kyushu University

Nakano, Yutaka

University Farm, Faculty of Agriculture, Kyushu University

https://doi.org/10.5109/27358

出版情報: 九州大学大学院農学研究院紀要. 58 (2)，pp.281-285，2013-09. Faculty of Agriculture， Kyushu University

バージョン :

権利関係 : 


\title{
Napier's Constant, Imaginary Unit, Circular Constant and Some Natural Phenomena
}

\section{Masataka SHIMOJO* and Yutaka NAKANO ${ }^{1}$}

\author{
Laboratory of Regulation in Metabolism and Behavior, Division of Animal and Bioresource Sciences \\ Department of Bioresource Sciences, Faculty of Agriculture, Kyushu University, \\ Fukuoka 812-8581, Japan \\ (Received April 26, 2013 and accepted May 9, 2013)
}

\begin{abstract}
This study investigated relationships between some mathematical constants [Napier's constant $(e)$, imaginary unit $(\boldsymbol{i})$, circular constant $(\pi)$ ] and some natural phenomena. The results obtained were as follows. The equality between exponential function based on $e$ and Bondi $\mathrm{K}$-factor suggested hypotheses that both expressions required each other and exponential function was associated with the space-time continuum. The mathematical application of $v \rightarrow \infty$ to Bondi K-factor led to $\boldsymbol{i}$, where $\boldsymbol{i}$ was interpreted as something other than $v$ due to the definition of $v$ as $0 \leq v<c$ ( $c=$ speed of light in vacuum). This suggested a hypothesis that the role of $\boldsymbol{i}$, though the phenomenon related to $\boldsymbol{i}$ looked like the phenomenon related to the infinite velocity, was to prohibit the state of $c \leq v<\infty$ by taking the form of wave. Euler's identity was related with Bondi K-factor to the power of $\boldsymbol{i}$ where $v=c(\exp (2 \pi)-1) /(\exp (2 \pi)+1) \approx 0.996 c(\approx$ muon's velocity). Applying $v \rightarrow \infty$ to Bondi K-factor to the power of $\boldsymbol{i}$ gave ' $\boldsymbol{i}$ to the power of $\boldsymbol{i}$, a many-valued function that suggested numeral approximations to some physical constants. Some natural phenomena including metallic number and muon's velocity were related to $\pi$. It was suggested that $e, \boldsymbol{i}$ and $\pi$ were the basso continuo being played in some natural phenomena.
\end{abstract}

Key words: Bondi K-factor, circular constant, imaginary unit, Napier's constant, natural phenomena

\section{INTRODUCTION}

Exponential function based on Napier's constant is frequently used for the simulation of various natural phenomena including the growth of plants and animals. Shimojo and Nakano (2013) investigated relationships between exponential functions and some natural phenomena. Circular constant and imaginary unit are also important components of many simulation models.

The present study was designed to investigate relationships between some mathematical constants (Napier's constant, imaginary unit, circular constant) and some natural phenomena by giving additional explanations and information to the previous report (Shimojo and Nakano, 2013).

\section{THREE MATHEMATICAL CONSTANTS AND SOME NATURAL PHENOMENA}

\section{Exponential function, Bondi $\mathrm{K}$-factor and related problems}

As shown in reports (Bondi, 1964; Shimojo, 2011b, 2011c; Shimojo and Nakano, 2012, 2013), solving simultaneous equations in hyperbolic function and Lorentz factor gives expressions that connect exponential function to Bondi K-factor (1) or to Lorentz factor (2),

${ }^{1}$ University Farm, Faculty of Agriculture, Kyushu University, Kasuya, Fukuoka 811-2307

* Corresponding Author (E-mail: mshimojo@agr.kyushu-u.ac.jp) Part of this study was conducted at Agri-Bio Research Laboratory at Ito Campus, Kyushu University, Motooka, Fukuoka 819-0395

$$
\begin{aligned}
\exp ( \pm \theta) & =\sqrt{\frac{1 \pm v / c}{1 \mp v / c}}, \\
& =\frac{1}{\sqrt{1-(v / c)^{2}}} \cdot(1 \pm v / c)
\end{aligned}
$$

where $0 \leq \theta<\infty, v=$ velocity of matter, $c=$ speed of light in vacuum, $0 \leq v<c$, double-sign corresponds.

Expression (1) seems to suggest hypotheses that exponential function and Bondi $\mathrm{K}$-factor require each other and exponential function is associated with the spacetime continuum.

As shown in a report (Shimojo and Nakano, 2012), replacing $\pm \theta$ with $r t$ gives

$$
\exp (r t)=\sqrt{\frac{1+v / c}{1-v / c}}
$$

where $r=$ relative growth rate, $t=$ time.

One of the possible interpretations of expression (3) seems to suggest a hypothesis that when the weight or space shows an exponential increase $(r>0, t>0)$, there is a causal energy $(v>0)$. Expression $\exp (r t)$ is used to simulate the growth of an individual plant (Blackman, 1919) or animal (Brody, 1945).

By the way, the power series of $\exp (r t)$ is given by

$$
\exp (r t)=\sum_{\boldsymbol{k}=\mathbf{0}}^{\infty} \frac{(r t)^{\boldsymbol{k}}}{k !} .
$$


The definite integral of power series of $\exp (r t)$ with respect to time is given by

$$
\int_{0}^{t}\left(\sum_{k=0}^{\infty} \frac{(r t)^{k}}{k !}\right) d t=\frac{1}{r}\left[\sum_{k=1}^{\infty} \frac{(r t)^{k}}{k !}\right]_{0}^{t} .
$$

If based on the power series, then the conservation of the form of $\exp (r t)$ under its definite integral seems to suggest a mathematical field that causes the pair appearance of ' 1 ' and '-1' (Shimojo et al., 2004),

$$
\begin{aligned}
\frac{1}{r}\left[\sum_{k=1}^{\infty} \frac{(r t)^{k}}{k !}\right]_{0}^{t} & =\frac{1}{r}\left[\left(1+\sum_{k=1}^{\infty} \frac{(r t)^{k}}{k !}\right)-1\right]_{0}^{t} \\
& =\frac{1}{r}\left[\sum_{k=0}^{\infty} \frac{(r t)^{k}}{k !}-1\right]_{0}^{t}
\end{aligned}
$$

The numeral ' 1 ' seems to suggest a hypothetic condensation of many sets of complex number and its complex conjugate (Shimojo et al., 2004),

$$
1=\prod_{\boldsymbol{k}=1}^{m} \exp \left(\boldsymbol{i} \theta_{\boldsymbol{k}}\right) \cdot \exp \left(-\boldsymbol{i} \theta_{\boldsymbol{k}}\right)
$$

where $\boldsymbol{i}$ = imaginary unit.

The natural logarithm of expression (8) gives

$$
\begin{aligned}
0 & =\sum_{k=1}^{m}\left(\left(\boldsymbol{i} \theta_{\boldsymbol{k}}\right)+\left(-\boldsymbol{i} \theta_{\boldsymbol{k}}\right)\right) \\
& =\sum_{\boldsymbol{k}=1}^{m}\left(\exp \left(\boldsymbol{i}\left(\frac{1}{2}+2 n\right) \pi\right) \theta_{\boldsymbol{k}}+\exp \left(-\boldsymbol{i}\left(\frac{1}{2}+2 n\right) \pi\right) \theta_{\boldsymbol{k}}\right)
\end{aligned}
$$

where $n=$ set of all integers, $\pi=$ circular constant.

This seems to suggest a collapse of the hypothetic condensation (8), leading to the pair appearance of $\boldsymbol{i} \theta_{\boldsymbol{k}}$ and $-\boldsymbol{i} \theta_{\boldsymbol{k}}$ and the immediate pair disappearance of them. This is based on the principal value $(n=0)$. Does the many-valued property of complex functions $[\ln (1)=2 n \pi \boldsymbol{i}$, $\left.\pm \boldsymbol{i}\left(\theta_{\boldsymbol{k}}+2 n \pi\right), n \neq 0\right]$ seem to suggest a hypothesis that $2 n \pi \boldsymbol{i}$ plays a background role in this phenomenon?

If there are replacements (11) and (12) in expression (8), then does this seem to suggest a hypothetic condensation of matter and antimatter?

$$
\begin{aligned}
& \exp (\boldsymbol{i} \theta) \quad \rightarrow \quad A \exp \left(\boldsymbol{i} \frac{2 \pi}{h}(p x-E t)\right) \\
& \exp (-\boldsymbol{i} \theta) \rightarrow A \exp \left(-\boldsymbol{i} \frac{2 \pi}{h}(p x-E t)\right),
\end{aligned}
$$

where $A=$ amplitude, $p=$ momentum, $x=$ position, $E=$ energy, $h=$ Planck constant.

Those hypotheses suggested in this section will be severely criticized or disregarded.

\section{Limitation of the velocity of matter}

The velocity $(v)$ at which the matter moves is limited by $0 \leq v<c$, where $c$ is speed of light in vacuum. Since the state that is equal to or greater than $c$ does not exist, the phenomenon that looks like the phenomenon associated with $c \leq v<\infty$ is considered something other than $v$. This hypothesis will be severely criticized or disregarded.

\section{Mathematical application of $v \rightarrow \infty$ to Bondi K-factor and Lorentz transformation}

The mathematical application of $v \rightarrow \infty$ to Bondi $\mathrm{K}$-factor and Lorentz transformation breaks them and leads to expressions (13) (20) (Shimojo, 2011b, 2011c; Shimojo and Nakano, 2013),

$$
\begin{aligned}
& \lim _{v \rightarrow \infty}\left(\sqrt{\frac{1 \pm v / c}{1 \mp v / c}}\right)=\lim _{v \rightarrow \infty}\left(\sqrt{\frac{c / v \pm 1}{c / v \mp 1}}\right) \\
& =\sqrt{-1} \\
& =\boldsymbol{i} \\
& =\exp \left(\boldsymbol{i}\left(\frac{1}{2}+2 n\right) \pi\right) \\
& m^{\prime}=\frac{m}{\sqrt{1-(v / c)^{2}}}=\frac{m c / v}{\sqrt{(c / v)^{2}-1}} \rightarrow 0, \\
& E=\frac{m c^{2}}{\sqrt{1-(v / c)^{2}}}=\frac{m c^{3} / v}{\sqrt{(c / v)^{2}-1}} \rightarrow 0, \\
& p=\frac{m v}{\sqrt{1-(v / c)^{2}}}=\frac{m c}{\sqrt{(c / v)^{2}-1}} \rightarrow-\boldsymbol{i} m c, \\
& E^{2}=m^{2} c^{4}+p^{2} c^{2} \rightarrow m^{2} c^{4}+(-\boldsymbol{i} m c)^{2} c^{2}=0, \\
& x^{\prime}=\frac{x-v t}{\sqrt{1-(v / c)^{2}}}=\frac{c x / v-c t}{\sqrt{(c / v)^{2}-1}} \rightarrow \boldsymbol{i} c t, \\
& t^{\prime}=\frac{t-v x / c^{2}}{\sqrt{1-(v / c)^{2}}}=\frac{c t / v-x / c}{\sqrt{(c / v)^{2}-1}} \rightarrow \frac{\boldsymbol{i} x}{c},
\end{aligned}
$$

where $m$ and $m^{\prime}=$ mass of matter, $\boldsymbol{i}=$ imaginary unit.

These expressions seem to suggest the phenomenon in the world of imaginary numbers, where $\boldsymbol{i}$ is interpreted as something other than $v$ because $\boldsymbol{i}$ is given by applying $v \rightarrow \infty$ to Bondi $\mathrm{K}$-factor. The mutually exclusive relationship between $\boldsymbol{i}$ and $v$ seems to suggest the mutually 
exclusive relationship between wave and matter. Does this seem to suggest a hypothesis that the role of $\boldsymbol{i}$, though the phenomenon related to $\boldsymbol{i}$ looks like the phenomenon related to the infinite velocity, is to prohibit the state of $c \leq v<\infty$ by taking the form of wave? If so, does $\boldsymbol{i}$ seem to suggest a hypothetic association with the simultaneous existence of different states or the nonlocal correlation between states that exist apart? Those hypotheses suggested in this section will be severely criticized or disregarded.

\section{Euler's formula and Bondi K-factor to the power of $i$}

As shown in the previous report (Shimojo and Nakano, 2013), extending expression (1) into complex numbers (21) gives expressions that connect Euler's formula with Bondi K-factor to the power of $\boldsymbol{i}$ (22) or with Lorentz factor to the power of $\boldsymbol{i}$ (23),

$$
\begin{aligned}
\exp ( \pm \boldsymbol{i} \theta) & =\cos ( \pm \theta)+\boldsymbol{i} \sin ( \pm \theta) \\
& =\left(\sqrt{\left.\frac{1 \pm v / c}{1 \mp v / c}\right)^{i}}\right. \\
& =\left(\frac{1}{\sqrt{1-(v / c)^{2}}} \cdot(1 \pm v / c)\right)^{\boldsymbol{i}}
\end{aligned}
$$

Expression (22) seems to suggest a hypothetic relationship between wave and Bondi $\mathrm{K}$-factor in the world of complex numbers. Shimojo et al. (2003a) applied the symmetric property of Euler's formula under its differentiation to the description of matter cycling in field-forageruminant relationships. Shimojo et al. (2003b) applied the stereographic representation of Euler's formula to the spiral form of micro-structures observed in plants and animals.

If expression (22) is related to replacements (11) and (12), then does expression (24) seem to suggest a hypothetic relationship between Bondi $\mathrm{K}$-factor to the power of $\boldsymbol{i}$ and wave function? In addition, do inequalities (27) and (28) seem to suggest a hypothetic difference between matter and antimatter?

$$
\begin{aligned}
& \left(\sqrt{\frac{1 \pm v_{\boldsymbol{d}} / c}{1 \mp v_{\boldsymbol{d}} / c}}\right)^{\boldsymbol{i}}=\exp \left( \pm \boldsymbol{i} \frac{2 \pi}{h}(p x-E t)\right), \\
& \ln \sqrt{\frac{1 \pm v_{\boldsymbol{d}} / c}{1 \mp v_{\boldsymbol{d}} / c}}= \pm \frac{2 \pi}{h}(p x-E t), \\
& = \pm \frac{2 \pi}{h}\left(\frac{h}{\lambda} x-h \nu t\right) \\
& -\frac{2 \pi}{h}(p x-E t)<0<\frac{2 \pi}{h}(p x-E t),
\end{aligned}
$$

$$
-\frac{2 \pi}{h}\left(\frac{h}{\lambda} x-h \nu t\right)<0<\frac{2 \pi}{h}\left(\frac{h}{\lambda} x-h \nu t\right),
$$

where $v_{\boldsymbol{d}}= \pm c(\exp ( \pm d)-1) /(\exp ( \pm d)+1), \quad d=4 \pi(p x-$ $E t) / h, d=4 \pi(h x / \lambda-h \nu t) / h, \ln =$ natural logarithm, $\lambda=$ wavelength, $\nu=$ frequency, double-sign corresponds.

As for Euler's identity that is a special case of Euler's formula,

$$
\begin{aligned}
& \exp ( \pm \boldsymbol{i} \pi)=-1 \\
& =\left(\sqrt{\frac{1 \pm v_{\pi} / c}{1 \mp v_{\pi} / c}}\right)^{i}, \\
& =\left(\frac{1}{\sqrt{1-\left(v_{\pi} / c\right)^{2}}} \cdot\left(1 \pm v_{\pi} / c\right)\right)^{i},
\end{aligned}
$$

where $v_{\pi}=c(\exp (2 \pi)-1) /(\exp (2 \pi)+1) \approx 0.996 c \approx$ muon's velocity.

Expressions (29) and (30) seem to suggest a hypothesis that Euler's identity is related with Bondi K-factor to the power of $\boldsymbol{i}$ where $v \approx$ muon's velocity. Those hypotheses suggested in this section will be severely criticized or disregarded.

\section{Mathematical application of $v \rightarrow \infty$ to Bondi K-factor to the power of $\boldsymbol{i}$}

The mathematical application of $v \rightarrow \infty$ to expression (22) gives expressions (32) (38),

$$
\begin{aligned}
& \lim _{v \rightarrow \infty}\left(\sqrt{\frac{1 \pm v / c}{1 \mp v / c}}\right)^{i}=\lim _{\boldsymbol{v} \rightarrow \infty}\left(\sqrt{\frac{c / v \pm 1}{c / v \mp 1}}\right)^{\boldsymbol{i}} \\
& =(\sqrt{-1})^{\boldsymbol{i}}=\boldsymbol{i}^{\boldsymbol{i}}, \\
& \pm \theta \quad \rightarrow \quad \ln \boldsymbol{i}=\boldsymbol{i}\left(\frac{1}{2}+2 n\right) \pi \\
& \exp ( \pm \boldsymbol{i} \theta) \rightarrow \boldsymbol{i}^{\boldsymbol{i}}, \\
& \boldsymbol{i}^{\boldsymbol{i}}=\cos (\ln \boldsymbol{i})+\boldsymbol{i} \sin (\ln \boldsymbol{i}), \\
& =\cos \left(\boldsymbol{i}\left(\frac{1}{2}+2 n\right) \pi\right)+\boldsymbol{i} \sin \left(\boldsymbol{i}\left(\frac{1}{2}+2 n\right) \pi\right) \\
& =\cosh \left(\left(\frac{1}{2}+2 n\right) \pi\right)-\sinh \left(\left(\frac{1}{2}+2 n\right) \pi\right) \\
& =\exp \left(-\left(\frac{1}{2}+2 n\right) \pi\right)
\end{aligned}
$$


where $\cosh =$ hyperbolic cosine , sinh $=$ hyperbolic sine. The mathematical application of $v \rightarrow \infty$ to Bondi K-factor to the power of $\boldsymbol{i}$ gives ' $\boldsymbol{i}$ to the power of $\boldsymbol{i}$ ' that takes real numbers. It takes one real number in response to one integer that $n$ takes. Does many-valued function (38) seem to suggest a hypothesis that ' $\boldsymbol{i}$ to the power of $\boldsymbol{i}$ ' takes two or more real numbers simultaneously unless $n$ takes one of the integers? Those hypotheses suggested in this section will be severely criticized or disregarded.

\section{Mathematical application of ' $i$ to the power of $i$ ' to some physical constants}

In spite of just playing with numbers based on the many-valued property of ' $\boldsymbol{i}$ to the power of $\boldsymbol{i}$ ', function (38) gives numeral approximations to some physical constants, apart from their units. Those are Newtonian constant of gravitation (39), speed of light in vacuum (40), Planck constant (41), Boltzmann constant (42), electron mass (43), proton mass (44), neutron mass (45) and Avogadro constant (46),

$$
\begin{aligned}
& 26.39823 \cdot \exp (-(1 / 2+2 \cdot 4) \pi) \\
& =26.39823 \cdot \boldsymbol{i}^{\boldsymbol{i}} \\
& \approx 6.67384 \cdot 10^{-11}=G\left(\mathrm{~m}^{3} \cdot \mathrm{kg}^{-1} \cdot \mathrm{s}^{-2}\right), \\
& 9.39184155 \cdot \exp (-(1 / 2+2 \cdot(-3)) \pi) \\
& =9.39184155 \cdot \boldsymbol{i}^{\boldsymbol{i}} \\
& \approx 299,792,458=c\left(\mathrm{~m} \cdot \mathrm{s}^{\mathbf{- 1}}\right), \\
& 1.77205281 \cdot \exp (-(1 / 2+2 \cdot 12) \pi) \\
& =1.77205281 \cdot \boldsymbol{i}^{\boldsymbol{i}} \\
& \approx 6.62606957 \cdot 10^{-34}=h(\mathrm{~J} \cdot \mathrm{s}), \\
& 0.4490483 \cdot \exp (-(1 / 2+2 \cdot 8) \pi) \\
& =0.4490483 \cdot \boldsymbol{i}^{\boldsymbol{i}} \\
& \approx 1.3806488 \cdot 10^{-\mathbf{2 3}}=k\left(\mathrm{~J} \cdot \mathrm{K}^{-\mathbf{1}}\right), \\
& 4.54942929 \cdot \exp (-(1 / 2+2 \cdot 11) \pi) \\
& =4.54942929 \cdot \boldsymbol{i}^{\boldsymbol{i}} \\
& \approx 9.10938291 \cdot 10^{-31}=e(\mathrm{~kg}), \\
& 15.599583411 \cdot \exp (-(1 / 2+2 \cdot 10) \pi) \\
& =15.599583411 \cdot \boldsymbol{i}^{\boldsymbol{i}} \\
& \approx 1.672621777 \cdot 10^{-27}=p(\mathrm{~kg}), \\
& 15.621086177 \cdot \exp (-(1 / 2+2 \cdot 10) \pi) \\
& =15.621086177 \cdot \boldsymbol{i}^{\boldsymbol{i}} \\
& \approx 1.674927351 \cdot 10^{-27}=n(\mathrm{~kg}), \\
& 0.80013773 \cdot \exp (-(1 / 2+2 \cdot(-9)) \pi) \\
& =0.80013773 \cdot \boldsymbol{i}^{\boldsymbol{i}} \\
& \approx 6.02214129 \cdot 10^{\mathbf{2 3}}=N_{\mathbf{A}}\left(\mathrm{mol}^{\mathbf{- 1}}\right) \text {. }
\end{aligned}
$$

Do these calculated approximations seem to suggest a hypothetic existence of ' $\boldsymbol{i}$ to the power of $\boldsymbol{i}$ ' at the back of those physical constants? There is a hypothesis that the weight of an individual plant or animal comes mainly from masses of proton and neutron. Those hypotheses suggested in this section will be severely criticized or disregarded.

\section{Some natural phenomena related to circular con- stant}

In the previous report (Shimojo and Nakano, 2013) a special relationship between Euler's formula, Bondi $\mathrm{K}$-factor and zeta function was shown through $\pi^{2} / 6$. This section adds some more natural phenomena to the previous report (Shimojo and Nakano, 2013) in order to show the relationship to $\pi$. Those are an example of Euler product (47), an example of zeta function (48), Euler's identity (49), metallic number multiplied by its conjugate (50), ' $\boldsymbol{i}$ to the power of $\boldsymbol{i}$ ' (51), an example of Bondi $\mathrm{K}$-factor (52) where $v=v_{\pi}(\approx$ muon's velocity) and uncertainty principle [(53), (54)],

$$
\begin{aligned}
& \pi=\sqrt{6 \prod_{p} \frac{p^{2}}{p^{2}-1}} \\
& =\sqrt{6 \sum_{k=1}^{\infty} \frac{1}{k^{2}}} \\
& =\frac{1}{1+2 n} \cdot \ln (-1)^{-i}, \\
& =\frac{1}{1+2 n} \cdot \ln \left(\frac{m+\sqrt{m^{2}+4}}{2} \cdot \frac{m-\sqrt{m^{2}+4}}{2}\right)^{-i} \\
& =\frac{-1}{1 / 2+2 n} \cdot \ln \left(\boldsymbol{i}^{\boldsymbol{i}}\right), \\
& =\ln \sqrt{\frac{1+v_{\pi} / c}{1-v_{\pi} / c}} \\
& \pi \geq \frac{h}{4 \cdot \Delta x \cdot \Delta p} \\
& \pi \geq \frac{h}{4 \cdot \Delta E \cdot \Delta t},
\end{aligned}
$$

where $p=$ prime numbers, $\left(m+\left(m^{2}+4\right)^{0.5}\right) / 2=$ metallic number, $v_{\pi}=c(\exp (2 \pi)-1) /(\exp (2 \pi)+1) \approx 0.996 c \approx$ muon's velocity.

Metallic number (50) gives golden number when $m=1$, silver number when $m=2$, and bronze number when $m=3$, respectively. Shimojo et al. (2011a) suggested, in a simple model of plant canopy, a hypothetic relationship between the golden number and the product of light extinction coefficient and leaf area index.

In addition, do expressions (55) and (56) seem to suggest a hypothetic relationship between $\pi$ and the product of wave components and the natural logarithm of Bondi K-factor? 


$$
\begin{aligned}
\pi & =\frac{h}{2(p x-E t)} \cdot \ln \sqrt{\frac{1+v_{\boldsymbol{d}} / c}{1-v_{\boldsymbol{d}} / c}} \\
& =\frac{h}{2(h x / \lambda-h \nu t)} \cdot \ln \sqrt{\frac{1+v_{\boldsymbol{d}} / c}{1-v_{\boldsymbol{d}} / c}}
\end{aligned}
$$

where $v_{\boldsymbol{d}}=c(\exp (d)-1) /(\exp (d)+1), d=4 \pi(p x-E t) / h$, $d=4 \pi(h x / \lambda-h \nu t) / h$.

Those hypotheses suggested in this section will be severely criticized or disregarded.

\section{Conclusions}

This study suggests that Napier's constant, imaginary unit and circular constant are the basso continuo being played in some natural phenomena.

\section{REFERENCES}

Blackman, V. H. 1919 The compound interest law and plant growth. Ann. Bot., 33: 353-360

Bondi, H. 1964 Relativity and Common Sense (Doubleday and Company Inc.,). Translation into Japanese by T. Yamanouchi for publication (1967), Kawade Shobo Publishers, Tokyo.

Brody, S. 1945 Time relations of growth of individuals and populations. In "Bioenergetics and Growth - with special reference to the efficiency complex in domestic animals", Reinhold
Publishing Corporation, New York, pp. 484-574

Shimojo, M., Y. Asano, K. Ikeda, R. Ishiwaka, T. Shao, H. Sato, M. Tobisa, Y. Nakano, N. Ohba, Y. Yano and Y. Masuda 2003a Complex representation of field-forage-ruminant relationships using symmetric properties of Euler's formula. J. Fac. Agr., Kyushu Univ., 47: 367-372

Shimojo, M., Y. Asano, K. Ikeda, R. Ishiwaka, T. Shao, H. Sato, M. Tobisa, Y. Nakano, N. Ohba, Y. Yano and Y. Masuda 2003b A stereographic representation of Euler's formula to show spirals and topological similarities to micro-structures in ruminants and forages. J. Fac. Agr., Kyushu Univ., 48: 71-75

Shimojo, M., K. Ikeda, R. Ishiwaka, H. Sato, Y. Asano, M. Tobisa, Y Nakano, N. Ohba, M. Eguchi and Y. Masuda 2004 Introducing complex numbers into basic growth functions - (II) Applying complex representation of ' $(-1)+1$ ' to definite integral of exponential function with base $e$ expanded into infinite series - . J. Fac. Agr., Kyushu Univ., 49: 337-341

Shimojo, M., M. Tobisa and Y. Nakano 2011a An application of the golden ratio and Fibonacci numbers to analyses of canopy structure of forage plants. J. Fac. Agr., Kyushu Univ., 56 $77-78$

Shimojo, M. 2011b Symmetry in motion in Euler's formula and its breakdown in hyperbolic function and growth function. $J$. Fac. Agr., Kyushu Univ., 56: 79-81

Shimojo, M. 2011c Exponential function, Bondi K-factor and imaginary unit. J. Fac. Agr., Kyushu Univ., 56: 285-286

Shimojo, M. and Y. Nakano 2012 Mathematical relationships between basic growth function and Bondi K-factor. J. Fac. Agr., Kyushu Univ., 57: 153-154

Shimojo, M. and Y. Nakano 2013 An investigation into relationships between exponential functions and some natural phenomena. J. Fac. Agr., Kyushu Univ., 58: 51-53 